\title{
STUDYING LEPTON FAMILY VIOLATION IN LEPTON-LEPTON COLLISIONS
}

\author{
V. V. Kabachenko and Yu. F. Pirogov \\ Theory Department, Institute for High Energy Physics, \\ Protvino, Moscow Region, RUSSIA \\ and \\ Moscow Institute of Physics and Technology, \\ Dolgoprudny, Moscow Region, RUSSIA
}

\begin{abstract}
In the context of the future high energy - high luminosity electron and muon colliders, all the relevant four-lepton processes with the lepton family violation (LFV) are systematically classified. The most general LFV effective lagrangians are found, and the helicity differential cross sections for the LFV processes are calculated. The six- and eightlepton Standard Model (SM) backgrounds are discussed, and the LFV processes clean of the six-lepton background are picked out. The possibility to suppress the six-lepton SM background, when present, by the unnatural initial beam polarizations is investigated. It is shown that the four-lepton LFV processes are amenable to experimental study in the lepton-lepton collisions in the most favourable cases up to the underlying scale of order $10^{2} \mathrm{TeV}$. Studying these processes should provide an essential part of the physics program for the next generation lepton colliders to unravel the outstanding flavour/family problem.
\end{abstract}


Introduction At present there are two principle to be solved problems in the Standard Model (SM): ( $i$ ) the spontaneous symmetry breaking problem, or that of origin of the electroweak gauge boson masses; $(i i)$ the flavour/family problem, or that of origin of the fermion masses and mixings. The former problem is superficially related with the TeV scale. It is expected to be mainly solved with the near future advent of the LHC, followed by the discovery and subsequent study of the Higgs boson. On the other hand, the latter problem should be related with a scale $\mathcal{O}(10 \mathrm{TeV})$ or higher and would require for its study the multi-TeV energy facilities.

These facilities may be provided by the future high energy - high luminosity $e^{+} e^{-}$and $e^{-} e^{-}$linear colliders [1], or $\mu^{+} \mu^{-}$[2] and possibly $\mu^{+} \mu^{+} / \mu^{-} \mu^{-}$ring colliders, or conceivably even $\mu^{+} e^{-}$and $\mu^{-} e^{-}$ring-linac colliders. It is not our aim to discuss the feasibility of all these projects. We would just like to emphasize that with the advent of such colliders a new era in the high energy physics, that of the unravelling the outstanding flavour/family problem, might set in.

The present-day theories give no convincing explanation to this obscure problem. So, an experimental key to solving it may possibly be given by studying the processes with family violation, in particular the lepton family violation (LFV), the latter being strictly forbidden in the SM. The lepton-lepton colliders provide the unique clean environment for studying LFV. We show that the advanced parameters of the colliders, namely $\sqrt{s} \geq 4 \mathrm{TeV}$ and integrated luminosity $L \geq 10^{3} \mathrm{fb}^{-1}$ conceivable at least for the $\mu \mu$ colliders, could allow one to study $\mathrm{LFV}$ in the four-lepton interactions up to the flavour/family scale $\mathcal{O}\left(10^{2} \mathrm{TeV}\right)$ which is quite assuring. We believe that this could provide an additional impetus in favour of lepton colliders.

Four-lepton LFV interactions Whatever the underlying nature of the flavour/family might be, at the energies much lower then the corresponding mass scale it should most probably manifest itself in the family violating contact interactions, in particular in the four-lepton interactions, too.

The SM invariant four-fermion operators are generically of the type $\bar{L} \bar{L} L L, \bar{R} \bar{R} R R, \bar{L} \bar{R} L R$, $\bar{L} \bar{L} R R$ and $\bar{R} \bar{R} L L$. Here $L$ and $R$ denote the left-handed and right-handed lepton fields, respectively. In this, it can be shown that the last two operators are forbidden for the processes with leptons exclusively. It follows thereof that all the allowed four-lepton operators can be reduced by the Fiertz rearrangement to the chirality conserving vector form

$$
\begin{aligned}
\mathcal{O}_{L L} & =\bar{L} \gamma_{\mu} L \bar{L} \gamma_{\mu} L, \\
\mathcal{O}_{R R} & =\bar{R} \gamma_{\mu} R \bar{R} \gamma_{\mu} R, \\
\mathcal{O}_{L R} & =\bar{L} \gamma_{\mu} L \bar{R} \gamma_{\mu} R,
\end{aligned}
$$

which we will use in what follows. Each of the fields in Eq. 1 can represent the lepton of any family, what in fact proliferates the number of independent operators. With this caveat in mind, the most general four-lepton effective lagrangian can be written as

$$
\mathcal{L}=\frac{1}{F^{2}} \sum_{i} \frac{c_{i}}{1+\delta_{i}} \mathcal{O}_{i},
$$

where $\delta_{i}=1$ for the operators with identical currents and $\delta_{i}=0$ for operators with different currents. Here $c_{i}$ are the generic free parameters, supposedly $c_{i}=\mathcal{O}(1)$, and $F$ is an unknown flavour/family violation mass scale ${ }^{2}$. Our expectation is that $F=\mathcal{O}\left(10^{2 \pm 1} \mathrm{TeV}\right)$.

\footnotetext{
${ }^{1}$ This form does not mean with necessity that all the underlying exchanges are of the vector type. They can equally well be transformed to the chirality violating scalar and tensor form. We use only vector type operators to fix somehow an ambiguity in the relative normalization of the different type operators.

${ }^{2}$ We take for the effective coupling constant $g_{\text {eff }}^{2}=1$. In the usual notations with $g_{\text {eff }}^{2}=4 \pi$, this would correspond to the scale $\Lambda=\sqrt{4 \pi} F$.
} 
There exist three possible types of the four-lepton LFV processes, namely $l^{\prime} l l l, l^{\prime} l^{\prime} l l$ and $l^{\prime \prime} l^{\prime} l l$. Here $l, l^{\prime}$ and $l^{\prime \prime}\left(l \neq l^{\prime} \neq l^{\prime \prime}\right)$ denote charged leptons/antileptons of different families. Let us consider these types of the LFV processes in turn.

(1) $\boldsymbol{l}^{\prime} \boldsymbol{l l l}$ type There are four independent operators in the effective lagrangian

$$
\mathcal{L}=\frac{1}{F^{2}}\left(\eta_{L L}\left(\bar{l}^{\prime} l\right)_{L}(\bar{l} l)_{L}+\eta_{L R}\left(\bar{l}^{\prime} l\right)_{L}(\bar{l} l)_{R}+(L \leftrightarrow R)+\text { h.c. }\right)
$$

where $(\overline{l l})_{L} \equiv \bar{l}_{L} \gamma_{\mu} l_{L}$, etc. Here and in what follows all free parameters $\eta$ etc are in general complex. Particular classes within the type, relevant for the electron and muon colliders, are $\mu e e e, e \mu \mu \mu$, $\tau e e e$ and $\tau \mu \mu \mu$.

(i) $\boldsymbol{\mu e}$ e Here one has $l^{\prime}=\mu, l=e$, and the relevant processes in this case are

$$
\begin{aligned}
& \text { (s) }\left\{\begin{array}{l}
e^{+} e^{-} \rightarrow \mu^{+} e^{-}, e^{+} \mu^{-}, \\
\mu^{+} e^{-} \rightarrow e^{+} e^{-},
\end{array}\right. \\
& \left(\begin{array}{l}
e^{-} e^{-} \rightarrow \mu^{-} e^{-}, \\
\mu^{-} e^{-} \rightarrow e^{-} e^{-} .
\end{array}\right.
\end{aligned}
$$

Here and in what follows it will be chosen as the $s$ channel that one with zero net electric charge $Q=0$, and as $u$ channel the one with $|Q|=2$. The proper differential cross section for the $s$ channel with unpolarized initial particles ist

$$
8 \pi s F^{4} \frac{d \sigma}{d \cos \theta}=\left(\eta_{L L}^{2}+\eta_{R R}^{2}\right) u^{2}+\frac{1}{4}\left(\eta_{L R}^{2}+\eta_{R L}^{2}\right)\left(s^{2}+t^{2}\right),
$$

where $s, t$ and $u$ are the usual Lorentz-invariant variables. In the $s$ channel one has $t=$ $-s(1-\cos \theta) / 2, u=-s(1+\cos \theta) / 2$. For definiteness, here and later on as the scattering angle $\theta$ there will be chosen that between the second particles in the initial and final pairs. The cross section for the $u$ channel corresponds to substitution $s \leftrightarrow u$ on the right hand side of Eq. (5).

The main background to these four-lepton LFV processes is given by the family conserving six-lepton SM processes with the same charged lepton content and with the imbalance of family compensated by a pair of corresponding neutrinos. More particularly, for the processes of Eq. (4) they are

$$
\begin{aligned}
e^{+} e^{-} & \rightarrow \mu^{+} e^{-}\left(\nu_{\mu} \bar{\nu}_{e}\right), e^{+} \mu^{-}\left(\bar{\nu}_{\mu} \nu_{e}\right) \\
\mu^{+} e^{-} & \rightarrow e^{+} e^{-}\left(\bar{\nu}_{\mu} \nu_{e}\right) \\
e^{-} e^{-} & \rightarrow \mu^{-} e^{-}\left(\bar{\nu}_{\mu} \nu_{e}\right) \\
\mu^{-} e^{-} & \rightarrow e^{-} e^{-}\left(\nu_{\mu} \bar{\nu}_{e}\right)
\end{aligned}
$$

We expect this background to be rather small due to the back-to-back requirement for charged final leptons, as well as the requirement for missing energy $E<2 \Delta E$, where $\Delta E$ is the energy uncertainty of charged final leptons. Nevertheless this background should be evaluated more thoroughly. Not knowing the characteristics of the detectors one can hardly do it. Hence the question arises as to whether this background, if present and dangerous, could be suppressed by using the polarized beams. The answer is that it can really be done so to a large extent. Let us consider this topic in more detail.

\footnotetext{
${ }^{3}$ Here and later on we do not consider separately the charge conjugate initial channels. The substitutions required for them are obvious.

${ }^{4}$ For simplicity we use here and in what follows only the real free parameters. For complex parameters one needs the obvious replacements $\eta^{2} \rightarrow|\eta|^{2}$ and so on, the interference terms being absent in the limit $m_{l} \ll \sqrt{s}$. Hence the $C P$ violating LFV processes in the lepton-lepton collisions at the high energies are suppressed.
} 
The typical Feynman diagrams for these background processes are given in Fig. 1. As it is seen, there are two topologically different classes of diagrams: those with and without the triple-boson vertex, one of the bosons in the vertex being neutral with necessity. On the other hand, the second class is divided into two subclasses: those with and without neutral boson exchange. The same classification takes place for the six-lepton SM background, if allowed, for all other LFV processes to be considered. It is clear that the neutral boson diagrams could be encountered only in processes with a particle and antiparticle of the same type, or with a going-through particle, as it is indeed the case with the $l^{\prime} l l l$ type of the LFV processes. These observations are important in discussing the possible background reduction by the initial state polarizations.

Suppose that arbitrary longitudinal polarization $P$ can be achieved. In this case, by proper choosing the "unnatural" polarization of one of the initial particles (i.e., $P>0$ for leptons, or $P<0$ for antileptons) one can suppress as $\Delta P \equiv 1-|P|$ all the background diagrams not containing the neutral bosons? What about the diagrams with the latter boson, they can be suppressed only if this boson is not coupled to the initial particles. But there are always diagrams with such a coupling and hence the background cannot be completely reduced by using just one polarized beam. Fortunately, by using the second polarized beam, again with the unnatural polarization, one can suppress all the left-out diagrams as well, but for the diagrams with the triple-boson vertex in the $s$ channel annihilation. The degree of the double polarization suppression will be just $\Delta P$ (instead of $\Delta P^{2}$ what might be expected a priori). Nevertheless the six-lepton SM background, if allowed, can rather efficiently be reduced in the bulk of the processes. More than this, there are "clean" processes without the six-lepton SM background at all (see later on)

The $\mu e e e$ class of the LFV processes is also unfavourable in another aspect. Indeed, one can show that

$$
B(\mu \rightarrow e e \bar{e})=\frac{1}{4}\left(\frac{v}{F}\right)^{4}\left(\eta_{L L}^{2}+\eta_{R R}^{2}+\frac{1}{2}\left(\eta_{L R}^{2}+\eta_{R L}^{2}\right)\right)
$$

with $v \equiv\left(\sqrt{2} G_{F}\right)^{-1 / 2}$ being the v.e.v. and $G_{F}$ being the Fermi constant. From】 $B(\mu \rightarrow e e \bar{e})<$ $10^{-12}$ it follows that $F>2^{3 / 4}\left(\eta_{L L}^{2}+\eta_{R R}^{2}+\left(\eta_{L R}^{2}+\eta_{R L}^{2}\right) / 2\right)^{1 / 4} 10^{2} \mathrm{TeV}$. So, one expects typically for the LFV processes of Eq. (4) that $\sigma<10^{-3} \mathrm{fb}$, and hence at $L=10^{3} \mathrm{fb}^{-1}$ one gets no more than 1 event/year. Hence this case is marginally observable and constitutes in a sense the worst case study. For the rest of the four-lepton LFV processes there are either no experimental restrictions at all or just rather mild ones, $F>\mathcal{O}(1 \mathrm{TeV})$. Hence all of these processes can in principle be reliably observed.

Before going to the next type of the LFV processes let us complete the discussion of the left-out classes of the l'lll type.

( $i$ ) $\boldsymbol{e} \boldsymbol{\mu} \boldsymbol{\mu} \boldsymbol{\mu}$ This class could contribute to the following LFV processes

$$
\begin{aligned}
& (s)\left\{\begin{array}{l}
\mu^{+} \mu^{-} \rightarrow e^{+} \mu^{-}, \mu^{+} e^{-}, \\
\mu^{+} e^{-} \rightarrow \mu^{+} \mu^{-},
\end{array}\right. \\
& (u) \quad\left\{\begin{array}{l}
\mu^{-} \mu^{-} \rightarrow e^{-} \mu^{-}, \\
e^{-} \mu^{-} \rightarrow \mu^{-} \mu^{-} .
\end{array}\right.
\end{aligned}
$$

\footnotetext{
${ }^{5}$ The problem with rotating the "natural" polarization of the produced muons to the required unnatural one can be hoped to be eventually solved.

${ }^{6}$ We consider as even less probable the six-lepton SM background with some of the charged leptons missed as, say, in $e^{+} e^{-} \rightarrow \mu^{+} e^{-}\left(\not^{+} \mu^{-}\right)$, etc, or the eight-fermion SM background with missing quarks as, say, $e^{+} e^{-} \rightarrow s \bar{s} \rightarrow \mu^{+} e^{-}\left(\nu_{\mu} \bar{\nu}_{e} \not \mu \not \mu\right)$, etc.

${ }^{7}$ All the available restrictions on the LFV decay branchings are in what follows from Ref. [3] and correspond to $90 \%$ C.L.
} 
All the preceding discussion of the $\mu e e e$ class is unchanged but for the absence of experimental restrictions on the scale $F$. Needless to say that here the coefficients $\eta$ in the counterpart of Eq. (5) are some new free parameters for each of the classes within the type. The same goes without saying in what follows.

(iii) тeee The relevant LFV processes are

$$
\begin{array}{ll}
\text { (s) } & e^{+} e^{-} \rightarrow \tau^{+} e^{-}, e^{+} \tau^{-}, \\
\text {(u) } & e^{-} e^{-} \rightarrow \tau^{-} e^{-} .
\end{array}
$$

The cross sections and background are similar to the $\mu e e e$ case. From the limit $B(\tau \rightarrow e e \bar{e})<$ $3 \cdot 10^{-6}$ it follows the mild restriction $F>\mathcal{O}(1 \mathrm{TeV})$.

(iv) $\boldsymbol{\tau} \boldsymbol{\mu} \boldsymbol{\mu} \boldsymbol{\mu}$ The LFV processes are

$$
\begin{aligned}
& \text { (s) } \quad \mu^{+} \mu^{-} \rightarrow \tau^{+} \mu^{-}, \mu^{+} \tau^{-}, \\
& \text {(u) } \mu^{-} \mu^{-} \rightarrow \tau^{-} \mu^{-},
\end{aligned}
$$

and from $B(\tau \rightarrow \mu \mu \bar{\mu})<1.9 \cdot 10^{-6}$ it follows the same restriction on $F$. Cross sections and background are as before. Altogether, these four classes of the LFV processes depend on 16 free parameters.

(2) $\boldsymbol{l}^{\prime} \boldsymbol{l}^{\prime} \boldsymbol{l} \boldsymbol{l}$ type The most general LFV effective lagrangian of this type is given by

$$
\mathcal{L}=\frac{1}{2 F^{2}}\left(c_{L L}\left(\bar{l}^{\prime} l\right)_{L}\left(\bar{l}^{\prime} l\right)_{L}+c_{L R}\left(\bar{l}^{\prime} l\right)_{L}\left(\bar{l}^{\prime} l\right)_{R}+(L \leftrightarrow R)+\text { h.c. }\right)
$$

with $c_{L R}=c_{R L}$. Here $1 / 2$ in front of the expression is introduced to account for the identity of currents. One encounters three particular classes: $\mu \mu e e, \tau \tau e e$ and $\tau \tau \mu \mu$.

(i) $\boldsymbol{\mu \mu e e}$ Here one has $l^{\prime}=\mu$ and $l=e$. These interactions contribute to the following LFV reactions

$$
\begin{aligned}
& \text { (s) } \quad \mu^{+} e^{-} \rightarrow e^{+} \mu^{-} \text {, } \\
& \text { (u) }\left\{\begin{array}{l}
e^{-} e^{-} \rightarrow \mu^{-} \mu^{-}, \\
\mu^{-} \mu^{-} \rightarrow e^{-} e^{-} .
\end{array}\right.
\end{aligned}
$$

They are intimately related with the muonium-antimuonium $(M \bar{M})$ conversion $\mu^{+} e^{-} \leftrightarrow e^{+} \mu^{-}$䧃, [5] and were discussed in the context of the multiplicative conservation laws in Refs. [6, 7]

The $s$ channel cross section is

$$
8 \pi s F^{4} \frac{d \sigma}{d \cos \theta}=\left(c_{L L}^{2}+c_{R R}^{2}\right) u^{2}+\frac{1}{2} c_{L R}^{2}\left(s^{2}+t^{2}\right),
$$

and that for the $u$ channel corresponds to the substitution $s \leftrightarrow u$ on the right hand side.

There is no six-lepton SM background for all these processes, as well as for all others of the same type. This is because $\left|\Delta F_{l, l^{\prime}}\right|=2$ for two different types of leptons $l, l^{\prime}$ cannot be compensated simultaneously just by emission of two additional neutrinos, at least four of them being required. So the leading SM background here is given by the eight-lepton processes

$$
\begin{aligned}
\mu^{+} e^{-} & \rightarrow e^{+} \mu^{-}\left(\nu_{e} \nu_{e} \bar{\nu}_{\mu} \bar{\nu}_{\mu}\right), \\
e^{-} e^{-} & \rightarrow \mu^{-} \mu^{-}\left(\nu_{e} \nu_{e} \bar{\nu}_{\mu} \bar{\nu}_{\mu}\right), \\
\mu^{-} \mu^{-} & \rightarrow e^{-} e^{-}\left(\bar{\nu}_{e} \bar{\nu}_{e} \nu_{\mu} \nu_{\mu}\right) .
\end{aligned}
$$

\footnotetext{
${ }^{8}$ Operators of the form $\left(\bar{l}^{\prime} l^{\prime}\right)(\bar{l} l)$ do not violate family and are omitted.

${ }^{9}$ The effective lagrangian of Eq. (11) is the most general one consistent with the SM invariance, and as such it can be used for the model independent analysis of the $M \bar{M}$ conversion.
} 
It can be shown that among the corresponding Feynman diagrams there always exist those with emission of the neutral gauge boson by the initial particles. Hence the eight-lepton background cannot be completely suppressed by using just one polarized beam, double polarization being required. But we expect this background not to be fatal because of the smallness of its cross section, as well as the difficulty to concentrate almost all the energy just in two of the six final particles.

What about the experimental restriction on this class of interactions, it can be inferred only from the limit $G_{M \bar{M}}<1.8 \cdot 10^{-2} G_{F}$ [8] on the four-fermion effective coupling for the $M \bar{M}$ conversion and corresponds to $F>\mathcal{O}(1 \mathrm{TeV})$. This still leaves a lot of room to investigate at the future high energy lepton colliders, the $\mu^{-} \mu^{-} / \mu^{+} \mu^{+}$collider with required advanced parameters being be most realistic among three conceivable ones.

(ii) $\boldsymbol{\tau} \tau \boldsymbol{e}$ e The only relevant LFV process in this class is

$$
e^{-} e^{-} \rightarrow \tau^{-} \tau^{-}
$$

There is neither six-lepton SM background nor experimental restrictions on $F$.

(iii) $\boldsymbol{\tau} \boldsymbol{\tau} \boldsymbol{\mu} \boldsymbol{\mu}$ The similar LFV process here is

$$
\mu^{-} \mu^{-} \rightarrow \tau^{-} \tau^{-}
$$

Everything is the same as in the preceding case This type of the LFV interactions depends altogether on 9 free parameters.

(3) $\boldsymbol{l}^{\prime \prime} \boldsymbol{l}^{\prime} \boldsymbol{l} \boldsymbol{l}$ type This is the most general type of the four-lepton LFV interactions. The general effective lagrangian here ist'

$$
\begin{aligned}
\mathcal{L}=\frac{1}{F^{2}} & \left(c_{L L}^{(1)}\left(\bar{l}^{\prime \prime} l\right)_{L}\left(\bar{l}^{\prime} l\right)_{L}+c_{L R}^{(1)}\left(\bar{l}^{\prime \prime} l\right)_{L}\left(\bar{l}^{\prime} l\right)_{R}\right. \\
& +c_{L L}^{(2)}\left(\bar{l}^{\prime \prime} l^{\prime}\right)_{L}(\bar{l} l)_{L}+c_{L R}^{(2)}\left(\bar{l}^{\prime \prime} l^{\prime}\right)_{L}(\bar{l} l)_{R} \\
& \left.+c_{L R}^{(3)}\left(\bar{l}^{\prime \prime} l\right)_{L}\left(\bar{l} l^{\prime}\right)_{R}+(L \leftrightarrow R)+\text { h.c. }\right)
\end{aligned}
$$

Three different classes are conceivable: $\tau \mu e e, \tau e \mu \mu$ and $e \mu \tau \tau$.

(i) $\boldsymbol{\tau} \boldsymbol{\mu e}$ e Here one has $l^{\prime \prime}=\tau, l^{\prime}=\mu, l=e$, and the following LFV processes are allowed

(s) $\quad e^{+} e^{-} \rightarrow \tau^{+} \mu^{-}, \mu^{+} \tau^{-}$,

(t) $\quad \mu^{+} e^{-} \rightarrow \tau^{+} e^{-}$,

(u) $\quad \mu^{-} e^{-} \rightarrow e^{-} \tau^{-}$,

as well as

$$
\begin{array}{ll}
\text { (s) } & \mu^{+} e^{-} \rightarrow e^{+} \tau^{-}, \\
\text {(u) } & e^{-} e^{-} \rightarrow \mu^{-} \tau^{-}
\end{array}
$$

\footnotetext{
${ }^{10}$ Note that the last two processes are supplementary to those of the $\mu \mu e e$ class w.r.t. the study of the multiplicative conservation laws. Were these laws exact, the processes of the $l^{\prime} l^{\prime} l l$ type could nevertheless be always allowed independent of the particular assignment of the multiplicative quantum numbers. On the other hand, the allowance of the various interactions of the $l^{\prime} l l l$ and $l^{\prime \prime} l^{\prime} l l$ types depends crucially on the latter choice. This makes it obligatory to investigate experimentally all the conceivable channels of the LFV processes.

${ }^{11}$ Operators of the form $\left(\bar{l}^{\prime \prime} l\right)_{L}\left(\bar{l} l^{\prime}\right)_{L}$ are Fiertz-equivalent to those $\left(\bar{l}^{\prime \prime} l^{\prime}\right)_{L}(\overline{l l})_{L}$, and $\left(\bar{l}^{\prime \prime} l\right)_{R}\left(\bar{l} l^{\prime}\right)_{R}$ to $\left(\bar{l}^{\prime \prime} l^{\prime}\right)_{R}(\overline{l l})_{R}$.
} 
The $s$ channel cross section for the processes of Eq. (18) is

$$
\begin{aligned}
32 \pi s F^{4} \frac{d \sigma}{d \cos \theta} & =\left(c_{L L}^{(2) 2}+c_{R R}^{(2) 2}\right) u^{2} \\
& +\left(c_{L R}^{(2) 2}+c_{R L}^{(2) 2}\right) t^{2}+\left(c_{L R}^{(3) 2}+c_{R L}^{(3) 2}\right) s^{2},
\end{aligned}
$$

the other two being obtained by substitutions, respectively, $s \leftrightarrow t$ and $s \leftrightarrow u$ on the right hand side. The cross section for the first process of Eq. (19) is

$$
8 \pi s F^{4} \frac{d \sigma}{d \cos \theta}=\left(c_{L L}^{(1) 2}+c_{R R}^{(1) 2}\right) u^{2}+\frac{1}{4}\left(c_{L R}^{(1) 2}+c_{R L}^{(1) 2}\right)\left(s^{2}+t^{2}\right),
$$

with that for the second process being obtained by substitution $s \leftrightarrow u$ on the right hand side.

The six-lepton SM background is allowed for the processes of Eq. (18) but is forbidden for those of Eq. (19). The latter fact is because it is impossible to compensate $\left|\Delta F_{l}\right|=2$ for leptons of the kind $l$, plus additional LFV $\left|\Delta F_{l^{\prime}, l^{\prime \prime}}\right|=1$ for $l^{\prime}$ and $l^{\prime \prime}$, just by emission of two neutrinos. Nevertheless the eight-lepton SM background with family conservation is as always allowed. Finally, from $B(\tau \rightarrow \mu e \bar{e}, \bar{\mu} e e)<6.8 \cdot 10^{-6}$ it follows that $F>\mathcal{O}(1 \mathrm{TeV})$.

(ii) $\boldsymbol{\tau} \boldsymbol{e} \boldsymbol{\mu} \boldsymbol{\mu}$ It corresponds to $l^{\prime \prime}=\tau, l^{\prime}=e, l=\mu$, and the relevant LFV processes here are

(s) $\quad \mu^{+} \mu^{-} \rightarrow \tau^{+} e^{-}, e^{+} \tau^{-}$,

(t) $\quad \mu^{+} e^{-} \rightarrow \mu^{+} \tau^{-}$,

(u) $\quad e^{-} \mu^{-} \rightarrow \mu^{-} \tau^{-}$

and

(s) $\quad \mu^{+} e^{-} \rightarrow \tau^{+} \mu^{-}$,

(u) $\quad \mu^{-} \mu^{-} \rightarrow e^{-} \tau^{-}$.

The cross sections and backgrounds are similar to the preceding case. From $B(\tau \rightarrow e \mu \bar{\mu}, \bar{e} \mu \mu)<$ $7.1 \cdot 10^{-6}$ it follows the same lower bound $F>\mathcal{O}(1 \mathrm{TeV})$.

(iii) $\boldsymbol{e} \boldsymbol{\mu} \boldsymbol{\tau} \boldsymbol{\tau}$ Here one has $l^{\prime \prime}=e, l^{\prime}=\mu$ and $l=\tau$. The processes of interest are

(s) $\quad \mu^{+} e^{-} \rightarrow \tau^{+} \tau^{-}$

and

$$
\text { (u) } \quad \mu^{-} e^{-} \rightarrow \tau^{-} \tau^{-}
$$

Eqs. (20) and (21) with the proper choice for invariant variables are relevant here, too. The six-lepton SM background to the process of Eq. (24) is as before. That one for the process of Eq. (25) is again absent, only the eight-lepton background being allowed. Besides, there are no experimental restrictions on this class of interactions 5 . This last type of the LFV processes contains a total of 30 free parameters.

\footnotetext{
${ }^{12}$ We considered everywhere only the SM background. It is beyond our scope to discuss the possible instrumental background due to faking one kind of particles by another, or caused, say, by cosmic rays, etc. Because of the extreme rarity of the LFV events every conceivable background should be taken into account eventually. Hence what we have found are the most optimistic estimates.
} 
Discussion and conclusion Our general classification of the four-lepton LFV processes is summarized in Table 1. In Table 2 we present the helicity differential cross sections for the fourlepton LFV processes in the units of $\left(8 \pi s F^{4}\right)^{-1}$ (the upper expressions in each of the cellars). Let us remind that the coefficients $\eta, c$ and $c^{(i)}$ for the $l^{\prime} l l l, l^{\prime} l^{\prime} l l$ and $l^{\prime \prime} l^{\prime} l l$ types of the LFV processes, respectively, differ for various classes of the processes. Nevertheless they are designated in the same manner for simplicity. As it should be clear from the preceding exposition, all four-lepton LFV processes depend in general on the grand total of 55 free parameters.

One can easily infer from the Table 2 which particular four-lepton LFV operators could be studied in each of the channels. In the SM background clean channels all the contributing interactions could be studied equally well. In the channels with the six-lepton SM background the interactions contributing to the helicity channels with the unnatural initial polarizations could be studied more reliably.

The lower expressions in each of the cellars of the Table 2 represent the reference number of the LFV events $n_{\lambda_{1} \lambda_{2}}$ in helicity channels at the proper collider with $\sqrt{s}=4 \mathrm{TeV}$ and $L=10^{3} \mathrm{fb}^{-1}$ for the flavour/family scale $F=10^{2} \mathrm{TeV}$. The real number of the events $N_{\lambda_{1} \lambda_{2}}$ scales with $F, \sqrt{s}$ and $L$ as $\left(10^{2} \mathrm{TeV} / F\right)^{4}(\sqrt{s} / 4 \mathrm{TeV})^{2}\left(L / 10^{3} \mathrm{fb}^{-1}\right) n_{\lambda_{1} \lambda_{2}}$. For the unpolarized beams one has $N=\sum N_{\lambda_{1} \lambda_{2}} / 4$. If one takes as an observability criterion that the number of LFV events in a channel is equal, say, to 10 then the LFV mass scale that can be reached in the channel is $F=(\sqrt{s} / 4 \mathrm{TeV})^{1 / 2}\left(L / 10^{3} \mathrm{fb}^{-1}\right)^{1 / 4}\left(n_{\lambda_{1} \lambda_{2}} / 10\right)^{1 / 4} 10^{2} \mathrm{TeV}$.

We conclude that the LFV processes are quite amenable to experimental study at the future generation lepton colliders in the most favourable cases up to the underlying scale $\mathcal{O}\left(10^{2} \mathrm{TeV}\right)$. Studying these processes to solve the outstanding flavour/family problem might provide raison d'être and the great challenge for the colliders.

Acknowledgements This work is supported in part by the RFBR under grant No. 96-0218122 and in part by the Competition Centre for Fundamental Natural Sciences under grant No. 95-0-6.4-21. For drawing the Feynman diagrams use is made of the DÜRER graphical package [9].

\section{References}

[1] Physics and Technology of the Next Linear Collider, BNL-52502 (1996).

[2] $\mu^{+} \mu^{-}$collider: A Feasibility Report, BNL-52503 (1996).

[3] Review of Particle Properties, Phys. Rev. D54, 249 (1996).

[4] B. Pontecorvo, Zh. Eksp. Teor. Fiz. 33, 549 (1957) [Sov. Phys. JETP 6, 429 (1958)].

[5] G. Feinberg and S. Weinberg, Phys. Rev. Lett. 6, 381 (1961); Phys. Rev. 123, 1439 (1961).

[6] S. L. Glashow, Phys. Rev. Lett. 6, 196 (1961).

[7] G. W. S. Hou, NTUTH-96-05 (1996) hep-ph/9605204.

[8] K. Jungman et al., presented at the 23rd INS Int. Symp. on Nuclear and Particle Physics with Meson Beams in the $1 \mathrm{GeV} / \mathrm{c}$ Region, Tokyo, March 15-18, 1995.

[9] A. V. Razumov and S. R. Slabospitsky, DÜRER - Graphics for $\mathrm{HT}_{\mathrm{E}} \mathrm{X}$ (unpublished). 


\begin{tabular}{|c|c|c|c|c|}
\hline Type & Class & Processes & Restriction & Background \\
\hline \multirow{4}{*}{$l^{\prime} l l l$} & неее & $\begin{array}{l}e^{+} e^{-} \rightarrow \mu^{+} e^{-}, e^{+} \mu^{-} \\
\mu^{+} e^{-} \rightarrow e^{+} e^{-} \\
e^{-} e^{-} \rightarrow \mu^{-} e^{-} \\
\mu^{-} e^{-} \rightarrow e^{-} e^{-}\end{array}$ & $\mathcal{O}\left(10^{2} \mathrm{TeV}\right)$ & \multirow{4}{*}{ Yes } \\
\hline & $е \mu \mu \mu$ & $\begin{array}{l}\mu^{+} \mu^{-} \rightarrow e^{+} \mu^{-}, \mu^{+} e^{-} \\
\mu^{+} e^{-} \rightarrow \mu^{+} \mu^{-} \\
\mu^{-} \mu^{-} \rightarrow \mu^{-} e^{-} \\
e^{-} \mu^{-} \rightarrow \mu^{-} \mu^{-}\end{array}$ & No & \\
\hline & 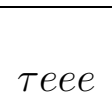 & $\begin{array}{l}e^{+} e^{-} \rightarrow \tau^{+} e^{-}, e^{+} \tau^{-} \\
e^{-} e^{-} \rightarrow \tau^{-} e^{-}\end{array}$ & $\mathcal{O}(1 \mathrm{TeV})$ & \\
\hline & $\tau \mu \mu \mu$ & $\begin{array}{l}\mu^{+} \mu^{-} \rightarrow \tau^{+} \mu^{-}, \mu^{+} \tau^{-} \\
\mu^{-} \mu^{-} \rightarrow \tau^{-} \mu^{-}\end{array}$ & $\mathcal{O}(1 \mathrm{TeV})$ & \\
\hline \multirow[t]{3}{*}{$l^{\prime} l^{\prime} l l$} & $\mu \mu е е$ & $\begin{array}{l}\mu^{+} e^{-} \rightarrow e^{+} \mu^{-} \\
e^{-} e^{-} \rightarrow \mu^{-} \mu^{-} \\
\mu^{-} \mu^{-} \rightarrow e^{-} e^{-}\end{array}$ & $\mathcal{O}(1 \mathrm{TeV})$ & \multirow[t]{3}{*}{ No } \\
\hline & $\tau \tau e e$ & $e^{-} e^{-} \rightarrow \tau^{-} \tau^{-}$ & $\mathrm{No}$ & \\
\hline & $\tau \tau \mu \mu$ & $\mu^{-} \mu^{-} \rightarrow \tau^{-} \tau^{-}$ & $\mathrm{No}$ & \\
\hline \multirow{6}{*}{$l^{\prime \prime} l^{\prime} l l$} & \multirow[t]{2}{*}{$\tau \mu e e$} & $\begin{array}{l}e^{+} e^{-} \rightarrow \tau^{+} \mu^{-}, \mu^{+} \tau^{-} \\
\mu^{+} e^{-} \rightarrow \tau^{+} e^{-} \\
\mu^{-} e^{-} \rightarrow e^{-} \tau^{-}\end{array}$ & $\mathcal{O}(1 \mathrm{TeV})$ & Yes \\
\hline & & $\begin{array}{l}\mu^{+} e^{-} \rightarrow e^{+} \tau^{-} \\
e^{-} e^{-} \rightarrow \mu^{-} \tau^{-}\end{array}$ & $\mathcal{O}(1 \mathrm{TeV})$ & No \\
\hline & \multirow[t]{2}{*}{$\tau е \mu \mu$} & $\begin{array}{l}\mu^{+} \mu^{-} \rightarrow \tau^{+} e^{-}, e^{+} \tau^{-} \\
\mu^{+} e^{-} \rightarrow \mu^{+} \tau^{-} \\
e^{-} \mu^{-} \rightarrow \mu^{-} \tau^{-}\end{array}$ & $\mathcal{O}(1 \mathrm{TeV})$ & Yes \\
\hline & & $\begin{array}{l}\mu^{+} e^{-} \rightarrow \tau^{+} \mu^{-} \\
\mu^{-} \mu^{-} \rightarrow e^{-} \tau^{-}\end{array}$ & $\mathcal{O}(1 \mathrm{TeV})$ & No \\
\hline & \multirow[t]{2}{*}{$\mu e \tau \tau$} & $\mu^{+} e^{-} \rightarrow \tau^{+} \tau^{-}$ & $\mathrm{No}$ & Yes \\
\hline & & $\mu^{-} e^{-} \rightarrow \tau^{-} \tau^{-}$ & $\mathrm{No}$ & $\mathrm{No}$ \\
\hline
\end{tabular}

Table 1 Summary of the four-lepton LFV processes (see text). 


\begin{tabular}{|c|c|c|c|c|c|}
\hline \multirow[t]{2}{*}{ Type } & \multirow[t]{2}{*}{ Processes } & \multicolumn{4}{|c|}{$\begin{array}{c}8 \pi s F^{4} d \sigma_{\lambda_{1} \lambda_{2}} / d \cos \theta \\
n_{\lambda_{1} \lambda_{2}} \\
\end{array}$} \\
\hline & & -- & ++ & -+ & +- \\
\hline \multirow{12}{*}{$l^{\prime} l l l$} & $e^{+} e^{-} \rightarrow \mu^{+} e^{-} / \tau^{+} e^{-}$ & $\eta_{R L}^{2} s^{2}$ & $\eta_{L R}^{2} s^{2}$ & $4 \eta_{R R}^{2} u^{2}+\eta_{L R}^{2} t^{2}$ & $4 \eta_{L L}^{2} u^{2}+\eta_{R L}^{2} t^{2}$ \\
\hline & $\mu^{+} \mu^{-} \rightarrow e^{+} \mu^{-} / \tau^{+} \mu^{-}$ & $5 \eta_{R L}^{2}$ & $5 \eta_{L R}^{2}$ & $1.7\left(4 \eta_{R R}^{2}+\eta_{L R}^{2}\right)$ & $1.7\left(4 \eta_{L L}^{2}+\eta_{R L}^{2}\right)$ \\
\hline & $e^{+} e^{-} \rightarrow e^{+} \mu^{-} / e^{+} \tau^{-}$ & $\eta_{L R}^{2} s^{2}$ & $\eta_{R L}^{2} s^{2}$ & $4 \eta_{R R}^{2} u^{2}+\eta_{L R}^{2} t^{2}$ & $4 \eta_{L L}^{2} u^{2}+\eta_{R L}^{2} t^{2}$ \\
\hline & $\mu^{+} \mu^{-} \rightarrow \mu^{+} e^{-} / \mu^{+} \tau^{-}$ & $5 \eta_{L R}^{2}$ & $5 \eta_{R L}^{2}$ & $1.7\left(4 \eta_{R R}^{2}+\eta_{L R}^{2}\right)$ & $1.7\left(4 \eta_{L L}^{2}+\eta_{R L}^{2}\right)$ \\
\hline & $\mu^{+} e^{-} \rightarrow e^{+} e^{-}$ & $\eta_{R L}^{2} s^{2}$ & $\eta_{L R}^{2} s^{2}$ & $4 \eta_{R R}^{2} u^{2}+\eta_{R L}^{2} t^{2 *}$ & $4 \eta_{L L}^{2} u^{2}+\eta_{L R}^{2} t^{2}$ \\
\hline & & $5 \eta_{R L}^{2}$ & $5 \eta_{L R}^{2}$ & $1.7\left(4 \eta_{R R}^{2}+\eta_{R L}^{2}\right)$ & $1.7\left(4 \eta_{L L}^{2}+\eta_{L R}^{2}\right)$ \\
\hline & $\mu^{+} e^{-} \rightarrow \mu^{+} \mu^{-}$ & $\eta_{L R}^{2} s^{2}$ & $\eta_{R L}^{2} s^{2}$ & $4 \eta_{R R}^{2} u^{2}+\eta_{R L}^{2} t^{2 *}$ & $4 \eta_{L L}^{2} u^{2}+\eta_{L R}^{2} t^{2}$ \\
\hline & & $5 \eta_{L R}^{2}$ & $5 \eta_{R L}^{2}$ & $1.7\left(4 \eta_{R R}^{2}+\eta_{R L}^{2}\right)$ & $1.7\left(4 \eta_{L L}^{2}+\eta_{L R}^{2}\right)$ \\
\hline & $e^{-} e^{-} \rightarrow \mu^{-} e^{-} / \tau^{-} e^{-}$ & $4 \eta_{L L}^{2} s^{2}$ & $4 \eta_{R R}^{2} s^{2 *}$ & $\eta_{L R}^{2} u^{2}+\eta_{R L}^{2} t^{2}$ & $\eta_{L R}^{2} t^{2}+\eta_{R L}^{2} u^{2}$ \\
\hline & $\mu^{-} \mu^{-} \rightarrow e^{-} \mu^{-} / \tau^{-} \mu^{-}$ & $20 \eta_{L L}^{2}$ & $20 \eta_{R R}^{2}$ & $1.7\left(\eta_{L R}^{2}+\eta_{R L}^{2}\right)$ & $1.7\left(\eta_{L R}^{2}+\eta_{R L}^{2}\right)$ \\
\hline & $\mu^{-} e^{-} \rightarrow e^{-} e^{-}$ & $4 \eta_{L L}^{2} s^{2}$ & $4 \eta_{R R}^{2} s^{2 *}$ & $\eta_{L R}^{2}\left(u^{2}+t^{2}\right)$ & $\eta_{R L}^{2}\left(u^{2}+t^{2}\right)$ \\
\hline & $e^{-} \mu^{-} \rightarrow \mu^{-} \mu^{-}$ & $10 \eta_{L L}^{2}$ & $10 \eta_{R R}^{2}$ & $1.7 \eta_{L R}^{2}$ & $1.7 \eta_{R L}^{2}$ \\
\hline \multirow{12}{*}{$l^{\prime \prime} l^{\prime} l l$} & $e^{+} e^{-} \rightarrow \tau^{+} \mu^{-}$ & $c_{R L}^{(3) 2} s^{2}$ & $c_{L R}^{(3) 2} s^{2}$ & $c_{R R}^{(2) 2} u^{2}+c_{L R}^{(2) 2} t^{2}$ & $c_{L L}^{(2) 2} u^{2}+c_{R L}^{(2) 2} t^{2}$ \\
\hline & $\mu^{+} \mu^{-} \rightarrow \tau^{+} e^{-}$ & $5 c_{R L}^{(3) 2}$ & $5 c_{L R}^{(3) 2}$ & $1.7\left(c_{R R}^{(2) 2}+c_{L R}^{(2) 2}\right)$ & $1.7\left(c_{L L}^{(2) 2}+c_{R L}^{(2) 2}\right)$ \\
\hline & $e^{+} e^{-} \rightarrow \mu^{+} \tau^{-}$ & $c_{L R}^{(3) 2} s^{2}$ & $c_{R L}^{(3) 2} s^{2}$ & $c_{R R}^{(2) 2} u^{2}+c_{L R}^{(2) 2} t^{2}$ & $c_{L L}^{(2) 2} u^{2}+c_{R L}^{(2) 2} t^{2}$ \\
\hline & $\mu^{+} \mu^{-} \rightarrow e^{+} \tau^{-}$ & $5 c_{L R}^{(3) 2}$ & $5 c_{R L}^{(3) 2}$ & $1.7\left(c_{R R}^{(2) 2}+c_{L R}^{(2) 2}\right)$ & $1.7\left(c_{L L}^{(2) 2}+c_{R L}^{(2) 2}\right)$ \\
\hline & $\mu^{+} e^{-} \rightarrow \tau^{+} e^{-}$ & $c_{R L}^{(2){ }^{2} s^{2}}$ & $c_{L R}^{(2) 2} s^{2}$ & $c_{R R}^{(2) 2} u^{2}+c_{L R}^{(3) 2} t^{2} *$ & $c_{L L}^{(2) 2} u^{2}+c_{R L}^{(3) 2} t^{2}$ \\
\hline & & $5 c_{R L}^{(2) 2}$ & $5 c_{L R}^{(2) 2}$ & $1.7\left(c_{R R}^{(2) 2}+c_{L R}^{(3) 2}\right)$ & $1.7\left(c_{L L}^{(2) 2}+c_{R L}^{(3) 2}\right)$ \\
\hline & $\mu^{+} e^{-} \rightarrow \mu^{+} \tau^{-}$ & $c_{L R}^{(2) 2} s^{2}$ & $c_{R L}^{(2) 2} s^{2}$ & $c_{R R}^{(2) 2} u^{2}+c_{L R}^{(3) 2} t^{2} *$ & $c_{L L}^{(2) 2} u^{2}+c_{R L}^{(3) 2} t^{2}$ \\
\hline & & $5 c_{L R}^{(2) 2}$ & $5 c_{R L}^{(2) 2}$ & $1.7\left(c_{R R}^{(2) 2}+c_{L R}^{(3) 2}\right)$ & $1.7\left(c_{L L}^{(2) 2}+c_{R L}^{(3) 2}\right)$ \\
\hline & $\mu^{+} e^{-} \rightarrow \tau^{+} \tau^{-}$ & $c_{R L}^{(3) 2} s^{2}$ & $c_{L R}^{(3) 2} s^{2}$ & $c_{R R}^{(2) 2} u^{2}+c_{R L}^{(2) 2} t^{2} *$ & $c_{L L}^{(2) 2} u^{2}+c_{L R}^{(2) 2} t^{2}$ \\
\hline & & $5 c_{R L}^{(3) 2}$ & $5 c_{L R}^{(3) 2}$ & $1.7\left(c_{R R}^{(2) 2}+c_{R L}^{(2) 2}\right)$ & $1.7\left(c_{L L}^{(2) 2}+c_{L R}^{(2) 2}\right)$ \\
\hline & $\mu^{-} e^{-} \rightarrow e^{-} \tau^{-}$ & $c_{L L}^{(2) 2} s^{2}$ & $c_{R R}^{(2) 2} s^{2} *$ & $c_{R L}^{(3) 2} u^{2}+c_{R L}^{(2) 2} t^{2}$ & $c_{L R}^{(3) 2} u^{2}+c_{L R}^{(2) 2} t^{2}$ \\
\hline & $e^{-} \mu^{-} \rightarrow \mu^{-} \tau^{-}$ & $5 c_{L L}^{(2) 2}$ & $5 c_{R R}^{(2) 2}$ & $1.7\left(c_{R L}^{(3) 2}+c_{R L}^{(2) 2}\right)$ & $1.7\left(c_{L R}^{(3) 2}+c_{L R}^{(2) 2}\right)$ \\
\hline
\end{tabular}

Table 2 Helicity differential cross sections for the LFV processes in the units of $\left(8 \pi s F^{4}\right)^{-1}$ (upper entries) and the representative number of events $n_{\lambda_{1} \lambda_{2}}$ in the various helicity channels at $F=10^{2} \mathrm{TeV}, \sqrt{s}=4 \mathrm{TeV}$ and $L=10^{3} \mathrm{fb}^{-1}$ (lower entries). (a) The processes with the six-lepton SM background. The asterisk $*$ marks the unnatural initial helicity channels without six-lepton SM background: the ++ helicity lepton-lepton collisions and the -+ helicity antilepton-lepton collisions (see text). 


\begin{tabular}{|c|l|c|c|c|c|}
\hline \multirow{3}{*}{ Type } & \multirow{2}{*}{ Processes } & \multicolumn{4}{|c|}{$8 \pi s F^{4} d \sigma_{\lambda_{1} \lambda_{2}} / d \cos \theta$} \\
\cline { 2 - 6 } & & -- & ++ & -+ & +- \\
\hline \multirow{5}{*}{$l^{\prime} l^{\prime} l l$} & $\mu^{+} e^{-} \rightarrow e^{+} \mu^{-}$ & $c_{L R}^{2} s^{2}$ & $c_{L R}^{2} s^{2}$ & $4 c_{R R}^{2} u^{2}+c_{L R}^{2} t^{2}$ & $4 c_{L L}^{2} u^{2}+c_{L R}^{2} t^{2}$ \\
& & $5 c_{L R}^{2}$ & $5 c_{L R}^{2}$ & $1.7\left(4 c_{R R}^{2}+c_{L R}^{2}\right)$ & $1.7\left(4 c_{L L}^{2}+c_{L R}^{2}\right)$ \\
\cline { 2 - 6 } & $e^{-} e^{-} \rightarrow \mu^{-} \mu^{-} / \tau^{-} \tau^{-}$ & $4 c_{L L}^{2} s^{2}$ & $4 c_{R R}^{2} s^{2}$ & $c_{L R}^{2}\left(u^{2}+t^{2}\right)$ & $c_{L R}^{2}\left(u^{2}+t^{2}\right)$ \\
& $\mu^{-} \mu^{-} \rightarrow e^{-} e^{-} / \tau^{-} \tau^{-}$ & $10 c_{L L}^{2}$ & $10 c_{R R}^{2}$ & $1.7 c_{L R}^{2}$ & $1.7 c_{L R}^{2}$ \\
\hline \multirow{5}{*}{$l^{\prime \prime} l^{\prime} l l$} & $\mu^{+} e^{-} \rightarrow e^{+} \tau^{-}$ & $c_{L R}^{(1) 2} s^{2}$ & $c_{R L}^{(1) 2} s^{2}$ & $4 c_{R R}^{(1) 2} u^{2}+c_{L R}^{(1) 2} t^{2}$ & $4 c_{L L}^{(1) 2} u^{2}+c_{R L}^{(1) 2} t^{2}$ \\
& $\mu^{+} e^{-} \rightarrow \tau^{+} \mu^{-}$ & $5 c_{L R}^{(1) 2}$ & $5 c_{R L}^{(1) 2}$ & $1.7\left(4 c_{R R}^{(1) 2}+c_{L R}^{(1) 2}\right)$ & $1.7\left(4 c_{L L}^{(1) 2}+c_{R L}^{(1) 2}\right)$ \\
\cline { 2 - 6 } & $e^{-} e^{-} \rightarrow \mu^{-} \tau^{-}$ & $4 c_{L L}^{(1) 2} s^{2}$ & $4 c_{R R}^{(1) 2} s^{2}$ & $c_{L R}^{(1) 2} t^{2}+c_{R L}^{(1) 2} u^{2}$ & $c_{L R}^{(1) 2} u^{2}+c_{R L}^{(1) 2} t^{2}$ \\
& $\mu^{-} \mu^{-} \rightarrow \tau^{-} e^{-}$ & $20 c_{L L}^{(1) 2}$ & $20 c_{R R}^{(1) 2}$ & $1.7\left(c_{L R}^{(1) 2}+c_{R L}^{(1) 2}\right)$ & $1.7\left(c_{L R}^{(1) 2}+c_{R L}^{(1) 2}\right)$ \\
\cline { 2 - 6 } & $\mu^{-} e^{-} \rightarrow \tau^{-} \tau^{-}$ & $4 c_{L L}^{(1) 2} s^{2}$ & $4 c_{R R}^{(1) 2} s^{2}$ & $c_{L R}^{(1) 2}\left(t^{2}+u^{2}\right)$ & $c_{R L}^{(1) 2}\left(t^{2}+u^{2}\right)$ \\
& & $10 c_{L L}^{(1) 2}$ & $10 c_{R R}^{(1) 2}$ & $1.7 c_{L R}^{(1) 2}$ & $1.7 c_{R L}^{(1) 2}$ \\
\hline
\end{tabular}

Table 2 Continued. (b) The processes clean of the six-lepton SM background (see text). 

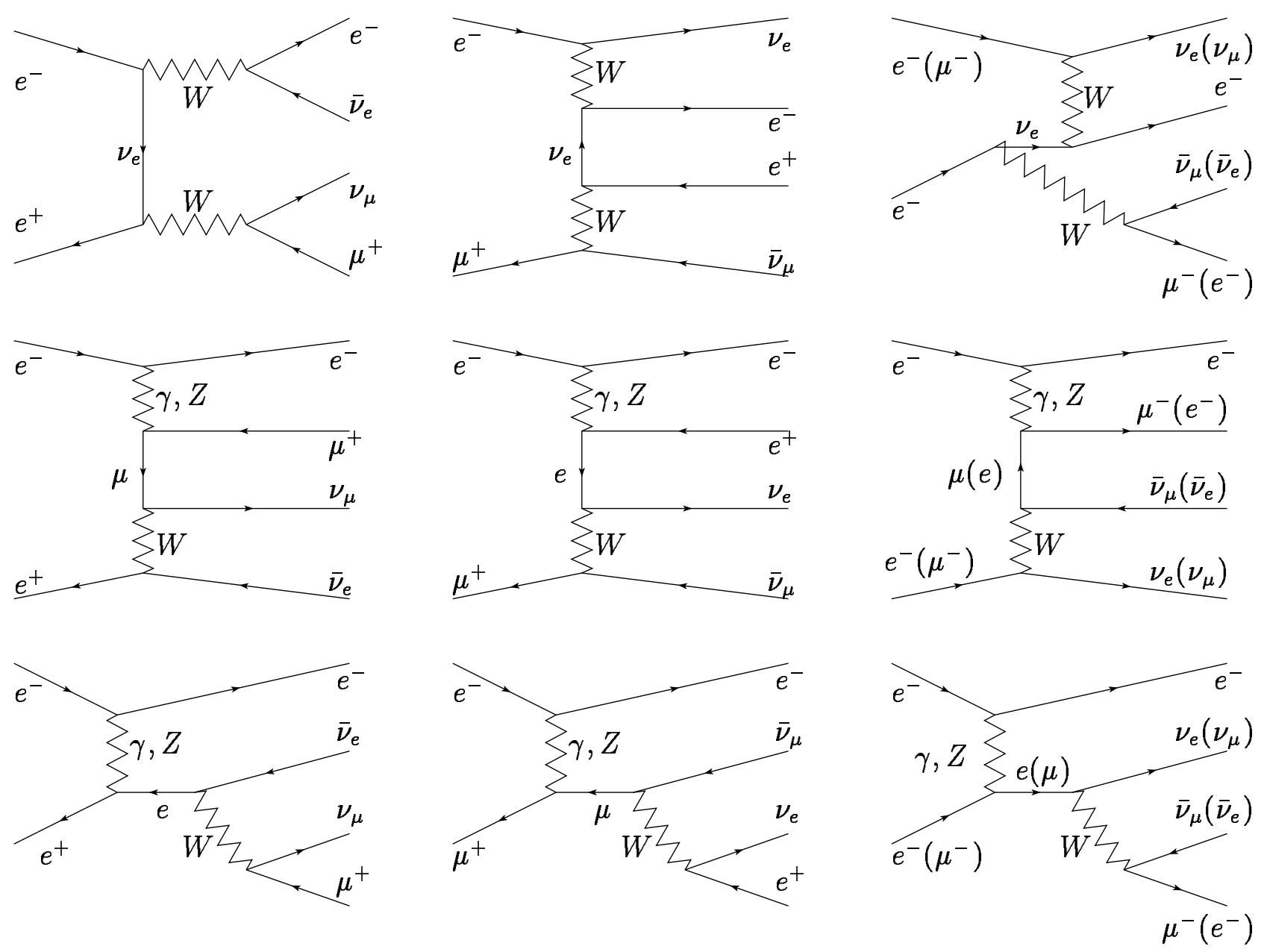

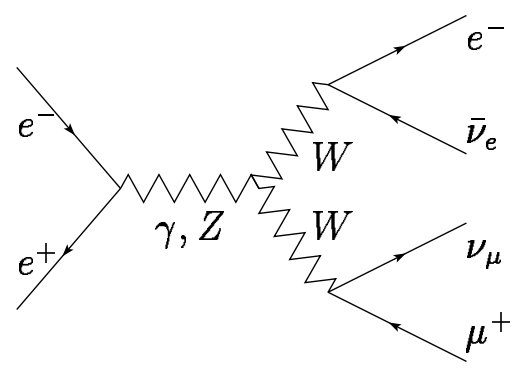

(a)

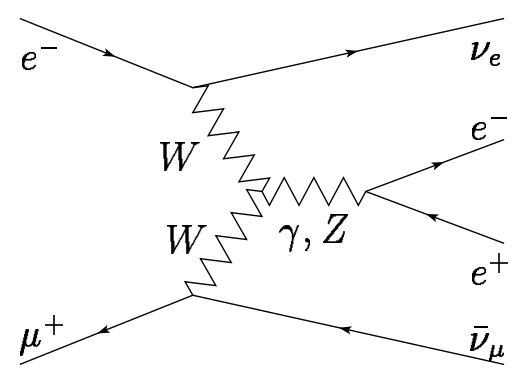

(b)

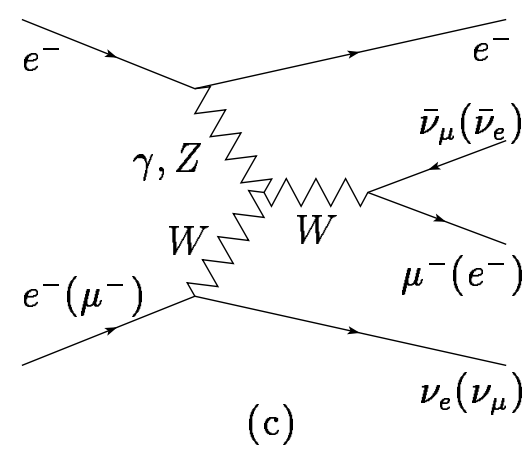

(c) 